\title{
Unbounded dynamics in dissipative flows: Rössler model
}

\author{
Roberto Barrio, ${ }^{1, a)}$ Fernando Blesa, ${ }^{2, b)}$ and Sergio Serrano ${ }^{1, c)}$ \\ ${ }^{1}$ Computational Dynamics Group, Dpto. Matemática Aplicada and IUMA, Universidad de Zaragoza, \\ E-50009 Zaragoza, Spain \\ ${ }^{2}$ Computational Dynamics Group, Dpto. Física Aplicada and IUMA, Universidad de Zaragoza, \\ E-50009 Zaragoza, Spain
}

(Received 14 October 2013; accepted 7 April 2014; published online 25 April 2014)

\begin{abstract}
Transient chaos and unbounded dynamics are two outstanding phenomena that dominate in chaotic systems with large regions of positive and negative divergences. Here, we investigate the mechanism that leads the unbounded dynamics to be the dominant behavior in a dissipative flow. We describe in detail the particular case of boundary crisis related to the generation of unbounded dynamics. The mechanism of the creation of this crisis in flows is related to the existence of an unstable focus-node (or a saddle-focus) equilibrium point and the crossing of a chaotic invariant set of the system with the weak-(un)stable manifold of the equilibrium point. This behavior is illustrated in the well-known Rössler model. The numerical analysis of the system combines different techniques as chaos indicators, the numerical computation of the bounded regions, and bifurcation analysis. For large values of the parameters, the system is studied by means of Fenichel's theory, providing formulas for computing the slow manifold which influences the evolution of the first stages of the orbit. C 2014 AIP Publishing LLC. [http://dx.doi.org/10.1063/1.4871712]
\end{abstract}

Unbounded dynamics is a relevant behavior in dynamical systems as it makes the orbits go far away. This phenomenon provides significant information in Hamiltonian systems, where it has been well studied (related to open Hamiltonian systems and chaotic scattering problems). Moreover, unbounded orbits in maps have been observed in many models, like the logistic and the Hénon map, and they have been studied both numerically and theoretically. In dissipative flows, unbounded dynamics appears mainly when the divergence of the flow is positive in unbounded regions, but it can be the dominant behavior or not. Moreover, when there are large regions with positive and negative divergence, chaotic invariants coexist with unbounded behavior. Therefore, to study the mechanism that creates this dominant behavior is a necessary task to improve the understanding of these dynamics. This goal is obtained by combining several state-of-theart numerical methods in the analysis of the Rössler model. Our results state that the key point in the transition to dominant unbounded dynamics is the existence of a focus (-saddle or -node) equilibrium point with a leading unstable manifold and a weak two dimensional (due to the focus) stable or unstable manifold that acts as a boundary of the escape region to infinity in the phase space. When the chaotic attractor or saddle of the system crosses this boundary, the unbounded dynamics becomes the dominant behavior. The numerical tests support this hypothesis and besides, they provide information of the organization of the parametric space in the region with dominant unbounded motion. The Fenichel's singular

\footnotetext{
a) Author to whom correspondence should be addressed. Electronic mail: rbarrio@unizar.es

b)fblesa@unizar.es

c) sserrano@unizar.es
}

perturbed theory provides theoretical insights about the influence of the slow-manifolds in the transient dynamics and the escape routes to infinity for large values of the parameters.

\section{INTRODUCTION}

The existence of unbounded dynamics is an important information of a system, and to describe the parametric dependence of such behavior is, therefore, a goal in the study of systems with such kind of dynamics. In Hamiltonian dynamics, this behavior is related to open nonintegrable Hamiltonian systems, like in chaotic scattering problems. In such a case, trajectories are launched in the scattering region; and if the system is open (i.e., there are some exits), some of these trajectories escape from this region in a direct way or present a transient chaotic behavior (related to the presence of an invariant, a chaotic saddle - a non attracting chaotic invariant set) in the phase space. ${ }^{1,2}$ The study of these systems has attracted the last few years a large number of researchers due to its relevance in practical applications as in Chemical Reaction Dynamics, Astrodynamics, Celestial Mechanics, Atomic and Nuclear Physics, Hydrodynamical processes, and so on (see the review paper $^{3}$ for a long list of applications and references).

A key point in the analysis of unbounded dynamics in dissipative systems is the study of the different crises leading to transient chaos (see the recent books of Refs. 2 and 4 and references therein). Most of the studies have been done for maps, ${ }^{5,6}$ focusing the attention into the crisis and the transient chaos phenomena. But the questions if later we have bounded or unbounded dynamics and why we have one or another kind of dynamics have not been studied in detail. Thus, one of the main objectives of this paper is to study the mechanism of 
creating a dominant unbounded dynamics for most of the phase space in flows. To that goal, we need to do a large number of numerical simulations, combining different techniques. A quite useful numerical technique is a fast chaos indicator, that is, a fast technique to detect chaotic and regular behavior (combined with a norm control in position variables to detect unbounded dynamics). In our case, we have used the OFLI2 chaos indicator ${ }^{7,8}$ (although any good chaos indicator may be used instead). This method is combined with the numerical study of the regions with bounded motion and with the study of bifurcation curves by means of numerical continuation techniques. All this information permits us to locate the main escape regions to infinity (regions in the parametric space where the unbounded dynamics dominates), to explain its appearance through a type of boundary crisis and to locate the areas inside these escape regions where some bounded motion persists. For large values of the parameters of the system, it is possible to use Fenichel's singular-perturbation theory to study the asymptotic behavior, also dominated by the unbounded dynamics but, for a compact domain, deeply influenced by the slow manifold of the system.

One requirement to have a dominant unbounded dynamics in a nonlinear chaotic flow is to have a positive divergence for some values of the parameters and variables of the system (in fact, it is necessary to have a positive divergence in unbounded regions of the phase space). Note that there may exist unbounded trajectories when the divergence of the system is negative: the initial volume eventually shrinks along the target trajectory, but the trajectory goes to infinity without bounds. But, in the negative case, unbounded orbits will not occur in large sets in the phase space (only in zero measure sets), contrary to what happens in the positive divergence case, where the unbounded motion is the normal behavior. So, for negative divergence values (globally dissipative systems), unbounded behavior is not relevant to the global dynamics of the system.

We are mainly interested in flows with a phase space that has regions with positive divergence and regions with negative divergence for the same values of the parameters. Therefore, in these systems, we may expect to have coexistence of unbounded and chaotic behavior (transient or not). Note that this situation occurs when the divergence of the system depends on some variables.

Within the field of dynamical systems and chaos for low dimensional systems, the Lorenz and the Rössler ${ }^{9}$ models are two classic chaotic problems that are heavily studied. Both of them have attracted a large number of studies and they continue to appear in the literature. The main reasons are that they are well-known but not completely understood, and they have become test problems for almost all new analytical and numerical techniques in computational dynamics. The Lorenz model is a closed system, that is, there is no unbounded dynamics as its divergence is negative for any value of the parameters (when all of them positive) and of the variables of the system. On the contrary, the sign of the divergence of the Rössler model depends on the value of some variables and parameters of the system. Thus, the Rössler model is an example of a flow with low dimensional chaos and unbounded dynamics. Therefore, we use this model as our test problem in this paper, but we remark that most of the results and simulations can be reproduced in most open dissipative flows.

This paper is organized as follows: Sec. II reviews some results of unbounded dynamics in maps. Section III studies regular and chaotic behavior and some bifurcations of the Rössler system and their influence in the organization of the space of parameters in the escape region to infinity. Besides, the mechanism of creation of the dominant unbounded dynamics is analyzed. In Sec. IV, the singular-perturbation Fenichel's theory is applied for large values of the parameters to study the asymptotic behavior of the system and its relation with the unbounded dynamics.

\section{UNBOUNDED DYNAMICS IN MAPS}

A phenomenon that frequently occurs in systems with a parametric dependence is related to a sudden change in the size or existence of chaotic attractors or saddles. This change is called crisis. This concept was introduced in Refs. 5 and 6, where also a classification into boundary and interior crises was done. Boundary crisis is related to the disappearance of the chaotic attractor, converted, for example, into a nonattracting chaotic invariant set. The effect of this crisis is a change in the dynamics of the system. A quite common mechanism that generates this crisis is related to the collision of the chaotic attractor with an unstable periodic orbit (PO) or fixed point, or with some of its manifolds.

The main objective of this section is to review the particular case of boundary crisis in simple maps related to the creation of a dominant unbounded motion. Note that this crisis has been already observed in literature but without pointing it out. To that goal, we just take two well-known maps. The first one is a one-dimensional quadratic map, related to the logistic map, given by

$$
x_{n+1}=C-x_{n}^{2} .
$$

This model ${ }^{5,10}$ has no fixed points for $C<-1 / 4$ and all orbits are unbounded (asymptotic to $-\infty$ ). At $C=-1 / 4$ a saddle-node bifurcation occurs and two fixed points (one stable and another unstable) are created. Later, the stable fixed point undergoes a cascade of period-doubling bifurcations to chaos. At $C=2$, the chaotic attractor suddenly disappears and all the orbits are unbounded. In fact, it is changed into a nonattracting chaotic invariant set, a chaotic saddle, due to the tangency between the chaotic attractor and the unstable fixed point ${ }^{2,5}$ (see Fig. 1). Therefore, following Grebogi, Ott, and Yorke, ${ }^{5}$ the system undergoes a boundary crisis. Moreover, at this value of the parameter $C$, the boundary crisis creates in this case a dominant unbounded behavior.

The second map is the classical Hénon map given by

$$
\begin{aligned}
& x_{n+1}=a+b y_{n}-x_{n}^{2}, \\
& y_{n+1}=x_{n} .
\end{aligned}
$$

This model was introduced by Hénon ${ }^{11}$ and now it is a wellknown map model. This model exhibits a chaotic attractor for several values of the parameters. At $a=-(1-b)^{2} / 4$, the system experiences a saddle-node bifurcation, where two 


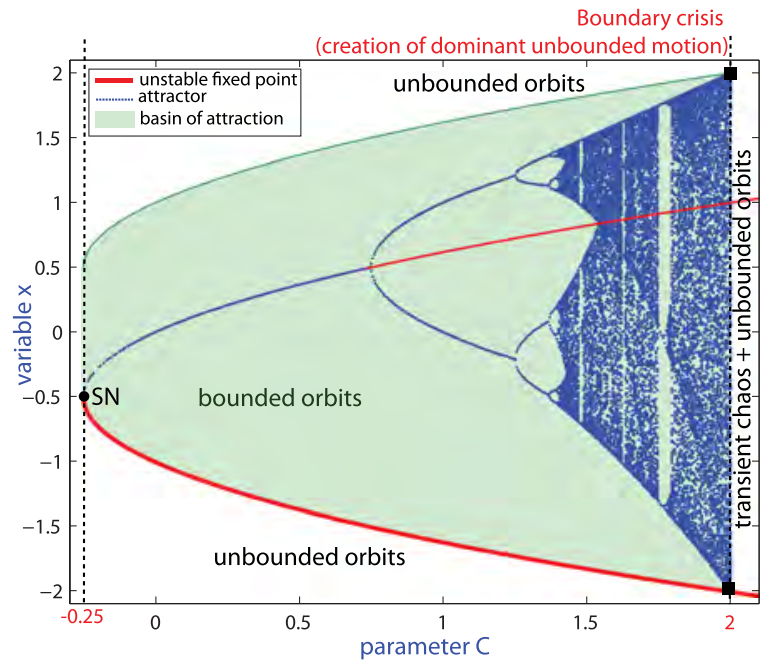

FIG. 1. Bifurcation diagram on the plane $(C, x)$ for the one-dimensional quadratic map.

fixed points, $p_{0}$ (stable) and $p_{1}$ (unstable), are created. Later, the stable fixed point undergoes a cascade of period-doubling bifurcations to chaos. A detailed study was performed in Ref. 12, where it was shown that, for $b=0.3$, a boundary crisis occurs at $a \approx 1.4269212$ due to a tangency between the stable manifold of the fixed point $p_{1}$ and the chaotic attractor. In Fig. 2, the invariants of the Hénon map are shown just before, at, and after the tangency of the chaotic attractor and the stable manifold of $p_{1}\left(W^{s}\left(p_{1}\right)\right)$. At this boundary crisis, the chaotic attractor disappears, becomes a chaotic saddle, and the unbounded motion prevails, which is the case that we are interested in analyzing in flows.

\section{RÖSSLER MODEL: BIFURCATIONS AND UNBOUNDED DYNAMICS}

In Sec. II, we have shown that in maps a particular case of boundary crisis, where at the same time a chaotic attractor disappears and the dominant behavior of the system becomes unbounded dynamics, is quite common. An interesting task is to see when and why this crisis also occurs in flows, situation that has not been studied in detail in the literature.

In this section, we analyze the Rössler model, a wellknown continuous dynamical system with low dimensional chaos and unbounded dynamics. The main goal here is to show the mechanism that generates the crisis from which the unbounded dynamics becomes the dominant one. To do it, we need to link the parametric space structure of the system (via bifurcation analysis to locate the key bifurcations) with the unbounded dynamics and the remaining bounded structures.

The Rössler equations ${ }^{9}$ are given by

$$
\begin{aligned}
& \dot{x}=-(y+z), \\
& \dot{y}=x+a y, \\
& \dot{z}=b+z(x-c),
\end{aligned}
$$

with $a, b, c \in \mathbb{R}$, and they are assumed to be positive and dimensionless. This model has two equilibrium points, $P_{1}$ and $P_{2}$, for $c^{2}>4 a b$, given by $P_{1}=\left(-a p_{1}, p_{1},-p_{1}\right)$ and $P_{2}=\left(-a p_{2}, p_{2},-p_{2}\right)$ with
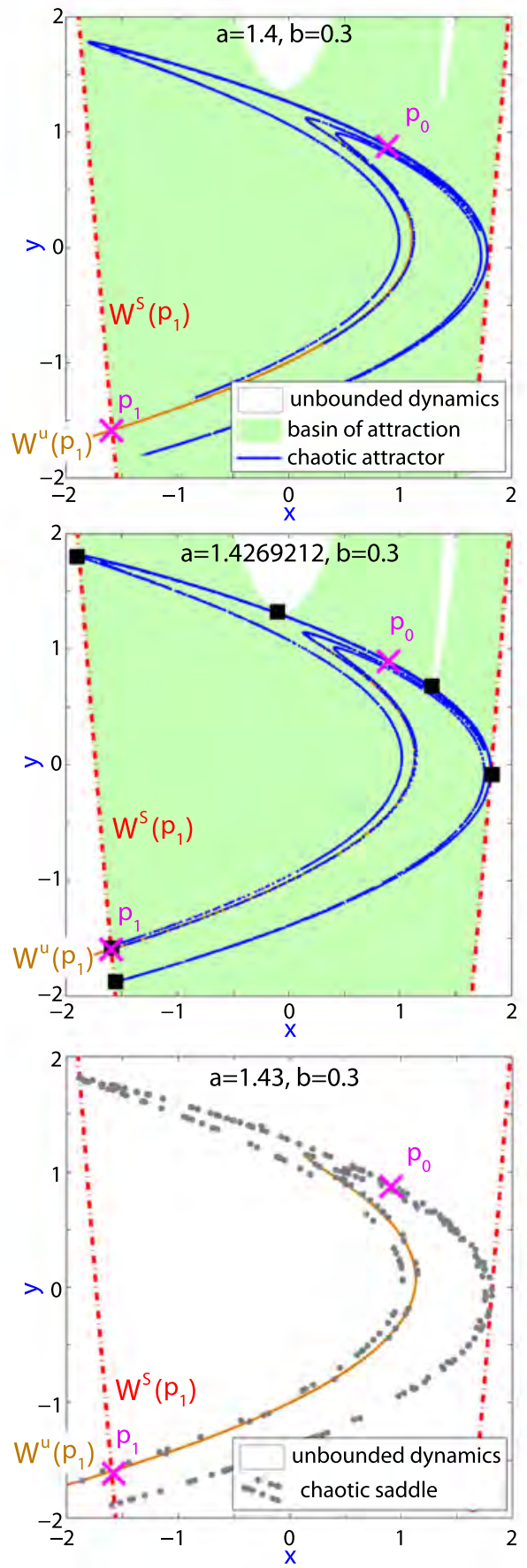

FIG. 2. Unstable fixed points $p_{0,1}$, and stable $\left(W^{s}\left(p_{1}\right)\right)$ and unstable manifolds $\left(W^{u}\left(p_{1}\right)\right)$ of the point $p_{1}$ of the Hénon map for $a=1.4, a=1.4269212$, and $a=1.43$, all cases with $b=0.3$. The black squares denote the tangency points.

$$
p_{1}:=-\frac{c+\sqrt{c^{2}-4 a b}}{2 a}, \quad p_{2}:=-\frac{c-\sqrt{c^{2}-4 a b}}{2 a} .
$$

Clearly, the condition of the existence of the equilibria $b_{\mathrm{E}} \equiv$ $b=c^{2} /(4 a)$ gives a surface of fold (saddle-node or tangent) bifurcations. These equilibria have several bifurcations, ${ }^{16,17}$ as different curves of Andronov-Hopf bifurcations around $P_{1}$ and $P_{2}$. These bifurcations give one mechanism of creation of limit cycles around equilibria. 
It is well known that for different sets of values of the parameters, the Rössler model exhibits a chaotic behavior, while for others a regular one. Besides, as its divergence is given by $\operatorname{div}\{\mathbf{f}(x, y, z)\}=a-c+x$, the Rössler system is not always dissipative. Thus, in a large region of parameters (especially when $a$ grows) and for large values of the variable $x$, we have a positive divergence and so unbounded orbits (note that unbounded orbits may also exist for negative divergence if the flow could diverge and expand to infinity in one direction while contracting in the perpendicular direction in such a way that the flow-divergence is negative). Therefore, apart from regular and chaotic orbits, the Rössler system also has unbounded orbits with a transient (chaotic or regular) behavior. This fact makes a theoretical and numerical analysis of this problem more difficult.

Hence, a study of the different regions in the parameter space will give some interesting results. In order to perform this study, in Refs. 13 and 14, the authors used a combination of different numerical techniques. To be precise, we used, as main techniques, the numerical continuation of bifurcation curves by means of the software AUTO, ${ }^{18}$ the numerical computation of regions with bounded attractors, and the fast chaos indicator OFLI2 (Refs. 7 and 8) (although any other chaos indicator would provide similar information). The combined use of these techniques permitted us to obtain an exhaustive numerical study of good quality in an affordable computational time.
Fig. 3 summarizes the global biparametric structure ${ }^{13-15}$ for $b=0.2$ : a codimension-2 Belyakov bifurcation point, ${ }^{19}$ in the homoclinic bifurcation curve of equilibrium $P_{2}$, is the key organizing point (top picture). It is the origin of three countable sets of subsidiary bifurcation curves: ${ }^{20}$ the first one of fold curves of periodic orbits; other set with period-doubling curves of POs and, finally, a set of saddle-focus homoclinic orbits. Fold- and cusp-shaped bifurcation curves of saddle-node periodic orbits, created at the Belyakov point, form the skeleton of a spiral structure joining shrimp shaped regular regions in the area with dominant bounded dynamics (see bottom picture). The study in Ref. 14 signs a universality of this structure in similar systems with chaotic attractors due to homoclinics of the Shilnikov saddle-focus ${ }^{14}$ and a relation with changes in the topology of the chaotic invariant sets of the system. ${ }^{15}$ Therefore, these bifurcation lines and points organize the parametric phase space inside the region where bounded dynamics is the dominant one. Besides, Andronov-Hopf bifurcations of $P_{1,2}$ equilibria and saddle-node bifurcations of equilibria complete the diagram, bounding regions with different type of equilibria. We will come back about this subject later.

Some questions arise from Fig. 3. In the white region above the saddle-node bifurcation curve, most of the orbits undergo unbounded motion. However, we can see that the Belyakov point and the sets of bifurcation curves starting

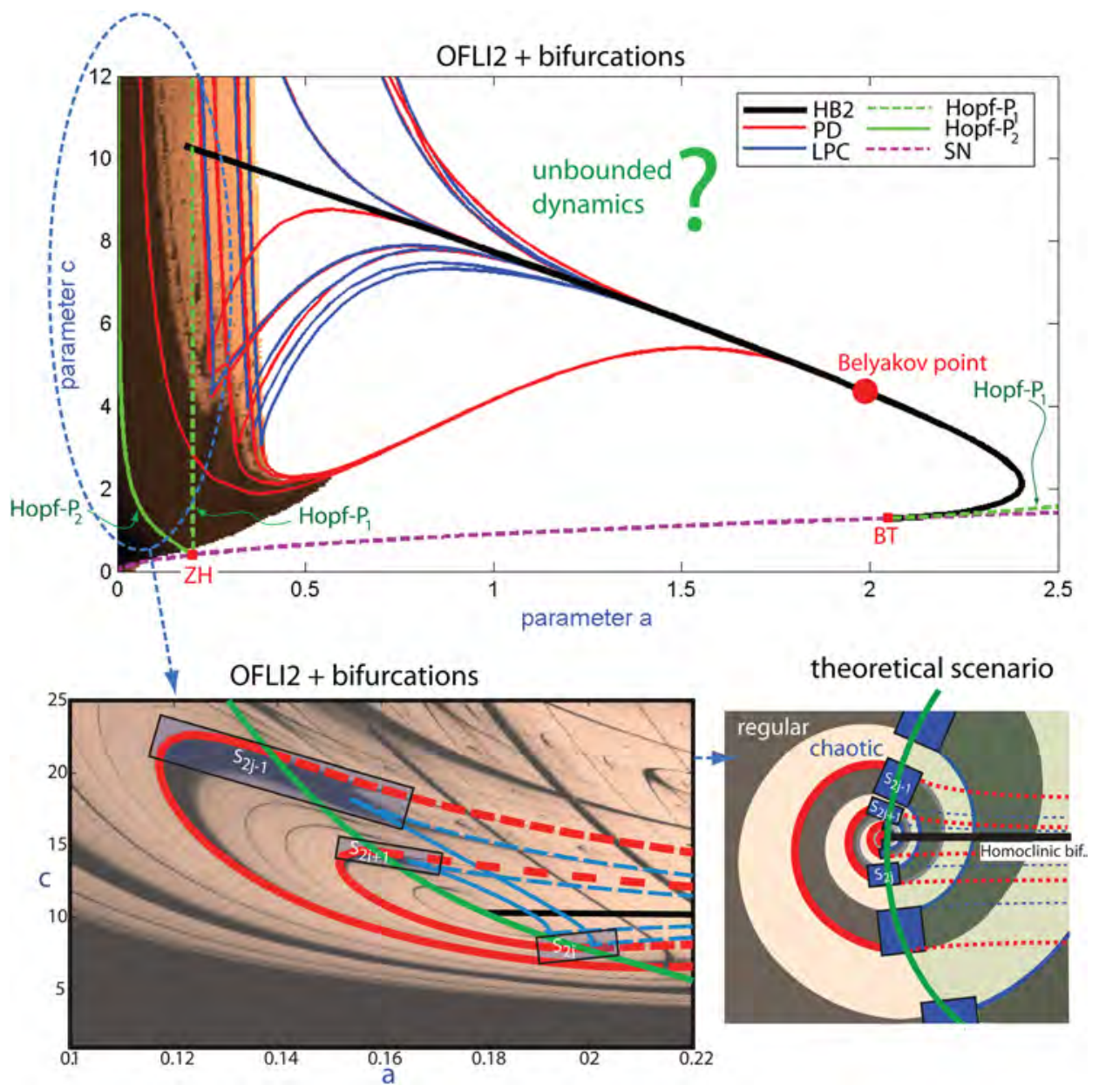

FIG. 3. Top: generation of structures from global bifurcations ${ }^{13-15}$ (HB2 homoclinic bifurcation line of equilibrium $P_{2}, \mathrm{PD}$ period-doubling bifurcation, LPC limit point cycle bifurcation-fold bifurcationHopf- $P_{1,2}$ Andronov-Hopf bifurcation of $P_{1.2}$ equilibria, $\mathrm{SN}$ saddle-node bifurcation of equilibria). Bottom: magnifications of the global structure outside the regions with dominant unbounded dynamics. In all the plots, white means unbounded behavior, dark color means bounded regular behavior, and light color chaotic behavior. 
from it are placed in this region. Then, what influence do these bifurcation curves of periodic orbits here? Moreover, the spiral structures shown at bottom picture (and others) appear quite frequently in the bounded dynamics regions. But, what structures remain in the unbounded dynamics region? And what is the origin of the boundary crisis that separates both behaviors? The next subsections provide answers to these and other related questions.

\section{A. Dependence on initial conditions}

A first point to study is to locate the region with unbounded dynamics, its boundary and its main structures, if any. To that goal, just the use of fast chaos indicators is not a good choice as these indicators do not distinguish unbounded from bounded motion. A simple and efficient tool is to combine a fast chaos indicator with the control, over time, of the distance of our orbit to a specific point. If this distance exceeds a certain threshold, the motion is considered unbounded and the integration is interrupted. The choice of the specific cutoff value is taken heuristically for each dynamical system. We will call this combined use BPD (Biparametric Phase Diagrams), as the procedure is designed mainly for biparametric plots.

In Fig. 4, we present the BPD diagrams on the $(a, c)$ plane (with $b=0.2$ ) for different values of the initial conditions. Different colors are associated with different behaviors for initial conditions considered. White color for unbounded motion; blue color for regular behavior; red and green colors for chaotic behavior. On the top pictures, we can see that some small islands of bounded motion appear on different areas in the region with dominant unbounded motion. This situation is shown more clearly on magnifications. Note that a similar picture for just one set of initial conditions was presented in Ref. 21; but in this paper, we intend to provide a complete explanation of this phenomenon. These magnifications show different positions and shapes of the islands for different initial conditions. In the bottom picture, we present a mixture of BPD diagrams with different initial conditions, giving priority to those that experience a bounded motion. A comparison between this picture and the top ones shows, mainly for $c<15$, that the limit zone which separates the region with unbounded motion from the regions with bounded one varies clearly. These coexistences of different behaviors on the Rössler system depending on the initial conditions have not been considered in previous studies. $^{21}$

Besides, the local bifurcations $\operatorname{curves}^{22}$ of limit cycles over the BPD diagram show that the islands appear around those bifurcations, giving a mechanism for the creation of these bands. This situation will be analyzed in Subsection III B.

If we fix the value of the parameters and vary the initial conditions, we can realize that regions with different behavior exist. In Fig. 5, we fix the parametric values $(a, b, c)=$ $(0.504,0.2,19.0)$ (red-dotted points on the right-down corner of magnifications of Fig. 4) and we show the region where the motion is bounded for different values of the initial coordinate $z$. It can be seen how the structure of the region changes with $z$ giving a 3D structure that determines the
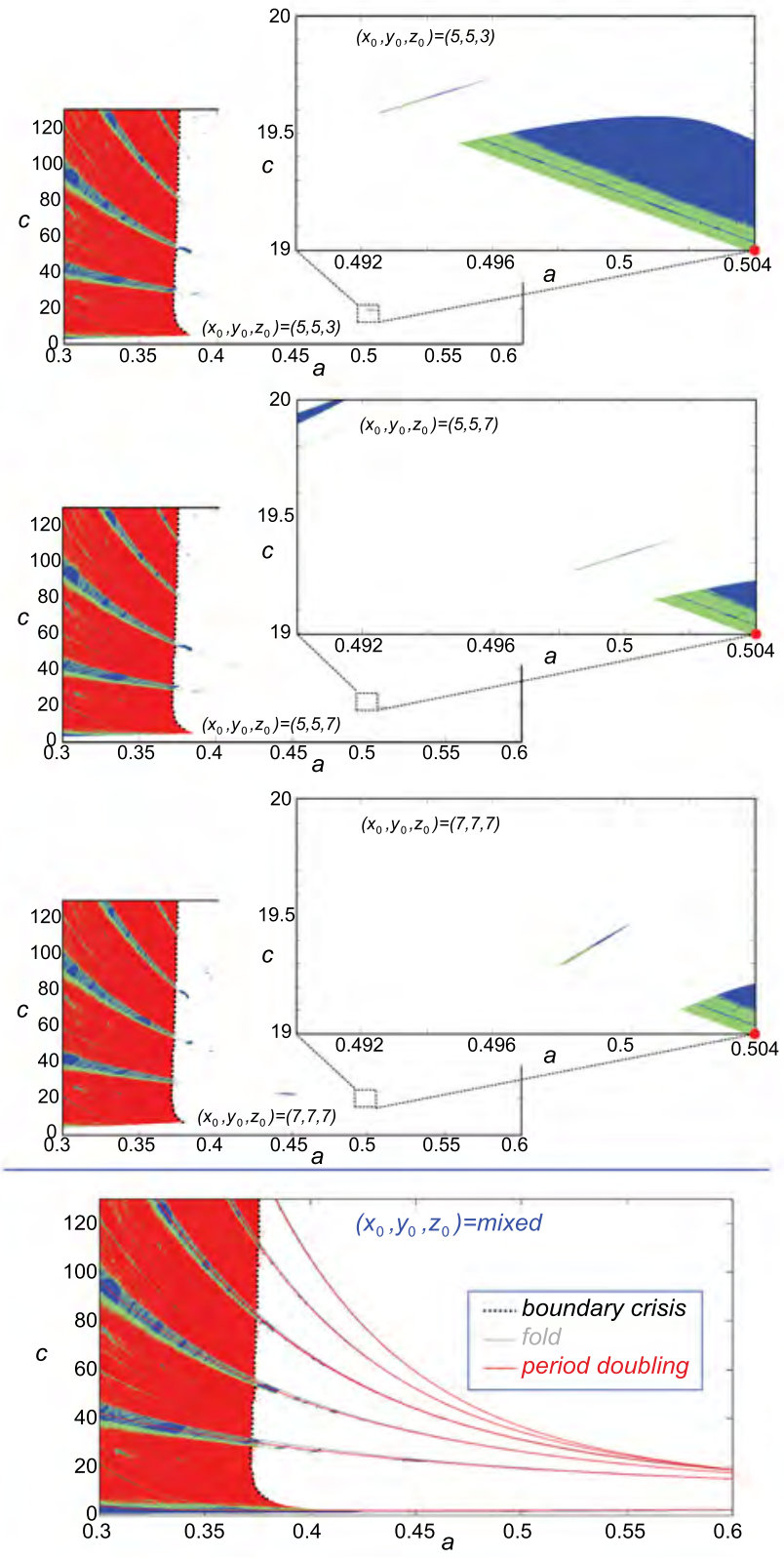

FIG. 4. Top: BPD diagrams on the $(a, c)$ plane $(b=0.2)$ for different values of the initial conditions and magnifications of some structures in the region with dominant unbounded dynamics. Bottom: bifurcation curves over BPD diagram.

basin of attraction of the bounded attractor. These plots show pictures with two spiral structures with bounded motion. This dependency on the initial conditions can be shown by plotting the orbits with different initial conditions. In the bottom pictures of Fig. 5, we provide two different orbits with the same values of parameters $((a, b, c)=(0.504,0.2,19.0))$ but with different initial conditions. The beginning of the orbit is plotted with red color, blue for the middle, and green for the last part. So, we can see that the first orbit escapes from any bounded region that we define, and the other orbit ends up converging to the attractor shown in red in Fig. 6 (it is in the basin of attraction of the chaotic attractor). Note that the basin of attraction has a fractal structure; and outside it, the unbounded dynamics dominates. 

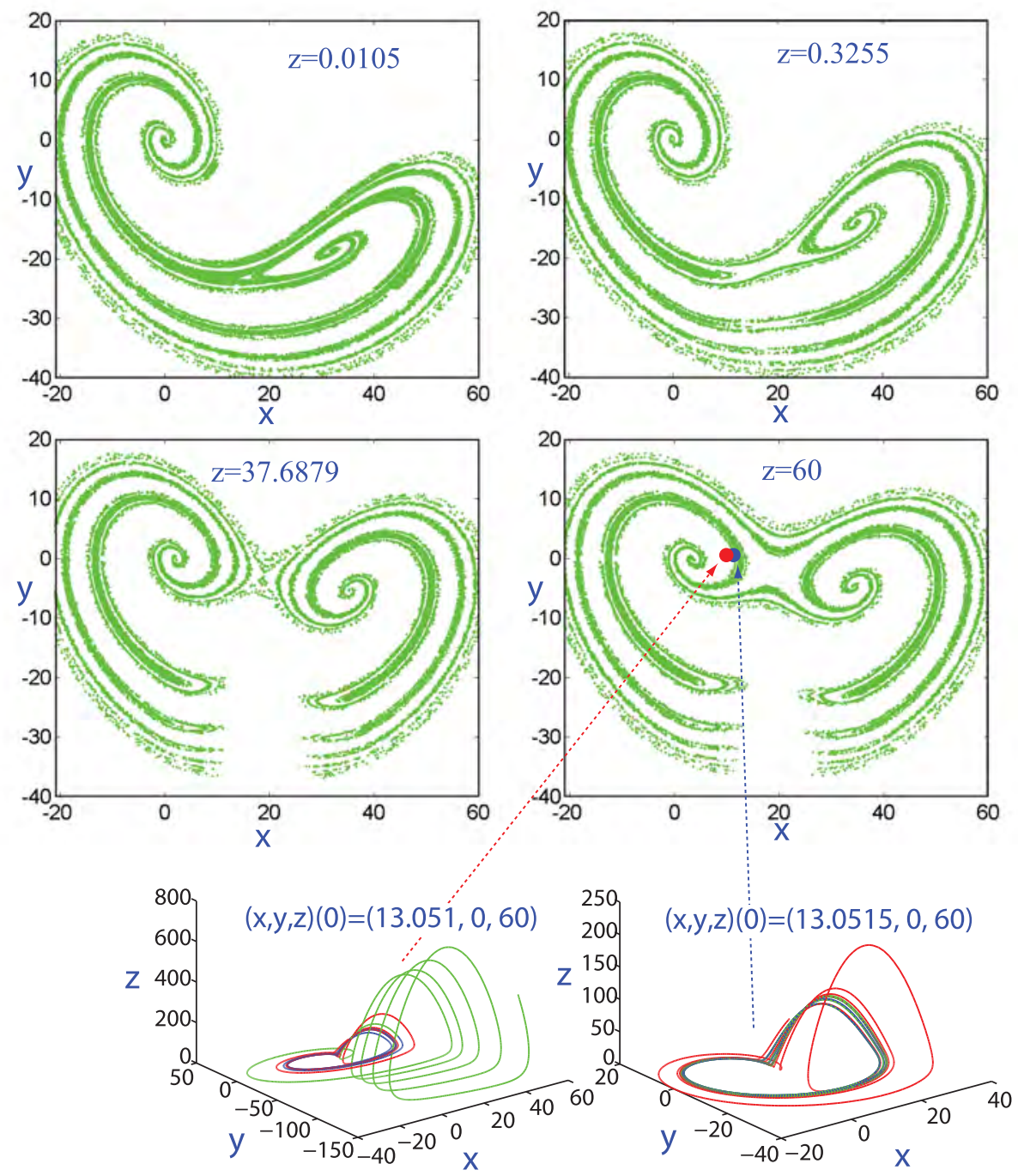

FIG. 5. Regions with bounded motion (sections of the fractal basin of attraction of the chaotic attractor) for different values of the initial condition $z$ (parametric values $(a, b, c)=(0.504,0.2,19.0))$. Bottom: two orbits with slightly different initial conditions. Left, unbounded orbit for initial conditions outside the fractal basin of the chaotic attractor. Right, bounded orbit converging to the red chaotic attractor in Fig. 6.
In order to visualize better the 3D structure of this basin of attraction, we show in Fig. 6 the three dimensional region with bounded motion for the same values of parameters than Fig. 5. Different colors are used just to distinguish between different sections with different values of $z$. The chaotic attractor appears in red. As we can see, the region with bounded motion goes shrinking when $z$ grows until around $z=950$, where it disappears. For negative values of $z$, part of the region disappears progressively when $z$ decreases. The basin of attraction of the chaotic attractor is much smaller than the region with unbounded motion and it is located close to the chaotic attractor (or stable periodic orbit) of the system. We can conclude that the basin of attraction is a bounded set with fractal structure inside the region with unbounded motion.

We have shown in Fig. 5 that, in the region with dominant unbounded dynamics, there are some islands where different behaviors coexist. If we look at the region not dominated by the unbounded dynamics, we might think that the same situation happens: Fig. 7 shows BPD diagrams for two sets of initial conditions, and we observe how both diagrams present the same basic structure of regular and chaotic motion, but they show differences of some "ghost bands" inside the regular region. In fact, these bands are generated by the transient dynamics of the orbits. What really we have is a stable limit cycle that is the attractor of the system inside the regular bands; but at the same time, the transient dynamics is dominated by the chaotic saddle of the system, what is shown on the bottom-left picture. On the right picture, we observe the attractor of the system in the regular band at the studied point. As we will see in Subsection IIIB, in the region with dominant unbounded motion, the transient dynamics generates a large orbit that enters in a region of the phase space that pushes the orbit far away; and therefore, this generates an unbounded orbit.

We remark that fast chaos indicators are quite useful techniques for detecting transient behavior due to the short final time of the simulations; but if we change the initial and final time, we can easily remove all the transient dynamics. Moreover, the combination of two or more sets of initial conditions, as we have done in Figure 7, permits us to detect transient dynamics or coexistence of different behaviors.

\section{B. Dependence on parameters}

In Subsection III A, we have located numerically where the change from bounded to unbounded motion occurs (the boundary crisis remarked in Fig. 4). In this subsection, we intend to provide some theoretical insights about this crisis.

As a first study, in Fig. 8, we show a similar BPD plot to that of Fig. 4, but now we remark the different regions of the 

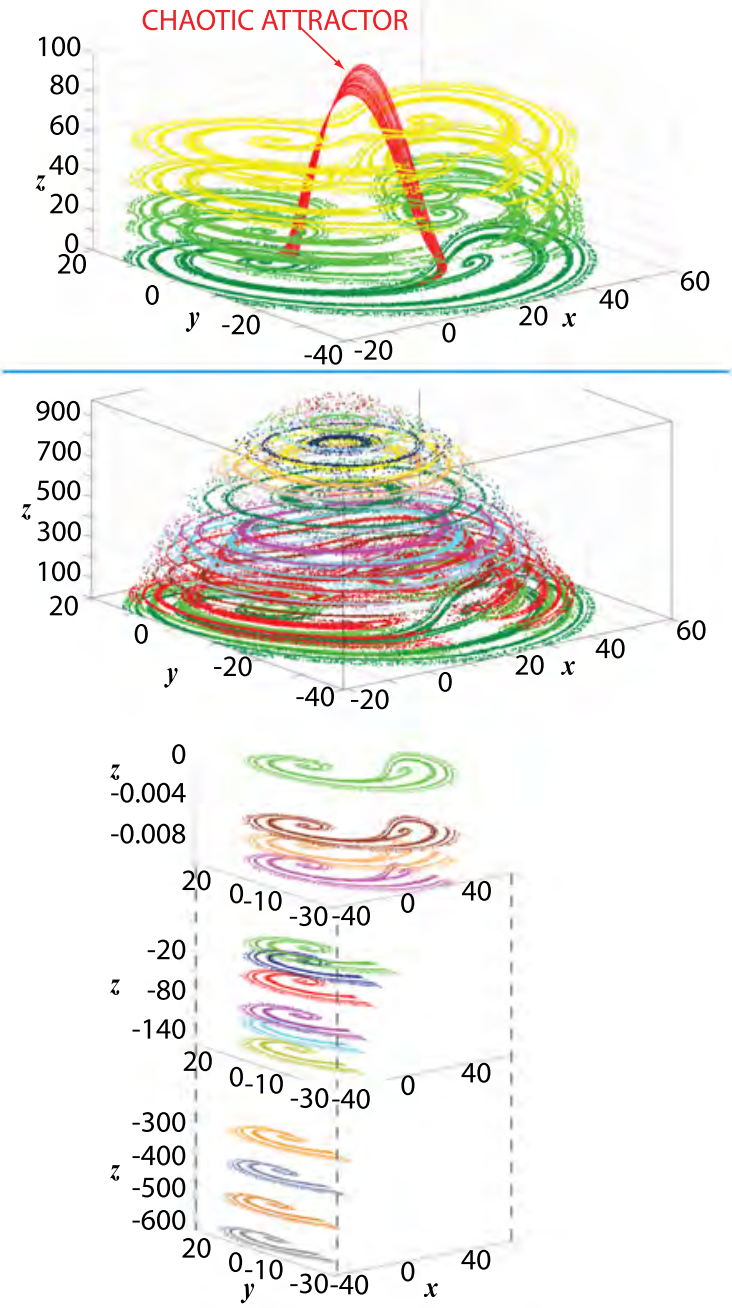

FIG. 6. Fractal basin of the chaotic attractor (in red) of the system for $(a, b, c)=(0.504,0.2,19.0)$.

parametric plane in function of the stability of the equilibrium points. At the same time, we plot some bifurcation lines, as the homoclinic bifurcation line of equilibrium $P_{2}$ and the fold and period-doubling bifurcations around it. In that parametric plane, there are six different regions, R1-R6. We observe from Fig. 8 that the crisis is not related to the local bifurcation lines, but it occurs in a region (R4) where the equilibrium point related to the creation of the unbounded dynamics $\left(P_{1}\right)$ is an unstable focus-node, what means that the unstable manifold has dimension 3 with two complex conjugate eigenvalues with a real part lower than the positive real eigenvalue. This implies that the equilibrium has a leading 1D strong-unstable manifold, which, as shown later, will have a relevant role in the unbounded dynamics. However, these regions do not give an explanation about the change of behavior. Therefore, a deeper analysis is necessary.

If we integrate two orbits with the same initial conditions for two slightly different sets of parameter values (the top black circles in Fig. 8), one on each side of the crisis curve, we can observe in Fig. 9 how for a while both trajectories behave similarly. However, at a certain point, the trajectory corresponding to the set of parameter values to the right of the boundary crisis goes far away.
The explanation of why, when, and where the second trajectory begins to diverge is given in Fig. 10. In this case, we plot several invariant manifolds of the equilibrium points. In Ref. 23, it has been established that, for any slow-fast dynamical system of low dimension, the location of the point where the curvature of the flow vanishes provides the analytical equation of the slow invariant manifold associated with such dynamical system. So, first we plot the slow-manifold of the system that generates several surfaces in the phase space. These surfaces are related to the regions where the motion is the slowest. But, the most relevant point in this study is the weak-unstable manifold of the equilibrium $P_{1}$. This weak-unstable manifold behaves as a real boundary between bounded and unbounded dynamics. That is, once a trajectory crosses outwards this manifold, the orbit is dominated by the leading 1D strong-unstable manifold that points far away. And, therefore, the orbit becomes an unbounded orbit. In the case that interests us, the boundary crisis occurs precisely when the chaotic attractor touches this manifold and crosses it. At this point, any orbit that follows the chaotic invariant set (now a chaotic saddle) leaves the transient chaotic behavior and it becomes an unbounded orbit. This mechanism generates the region with dominant unbounded motion and the limit in the parametric space is determined by the tangency among the weak-unstable manifold and the chaotic attractor. In Fig. 10, we plot, apart from the manifolds, the same orbits as in Fig. 9. Now, both behaviors are justified. Besides, we plot, for the non dominant unbounded dynamics, an orbit on the left (bounded orbit) and on the right (unbounded orbit) of the (linear) weak-unstable manifold. That is, for these values of the parameters (before the crisis), the bounded and unbounded motions coexist, depending on the initial conditions of the orbit, but the unbounded dynamics does not dominate in the region to the left of the weak-unstable manifold. On the contrary, after the crisis, not only the orbits on the right of the weak-unstable manifold diverge but also any orbit that follows for some time the chaotic-saddle. Then, most of the orbits diverge in this situation; and, therefore, now the unbounded dynamics dominates in the system.

We remark that in this model (and in general), we can observe boundary crises that lead to unbounded dynamics (the one studied in detail in this paper) and to bounded dynamics (just changing the character of the attractor). A remaining question is to study what bounded structures stay in the dominant unbounded dynamics region. In Subsection III A, we have seen that, around the period-doubling and fold bifurcation lines, there are some bands of bounded motion with a fractal basin of attraction; and outside them, the unbounded dynamics dominates. In Fig. 11, we show, on the top, the BPD of two regions in the parametric space, before and after the boundary crisis studied in this paper. In both regions, each regular band is originated by a fold bifurcation curve where two periodic orbits (one stable and the other unstable) arise. In plots A3 and B3, the stable periodic orbits in both regions are shown. Later, there is a chain of perioddoubling bifurcations that leads, through a Feigenbaum period-doubling cascade, to a "thin" chaotic attractor (A2 and B2). The region where this attractor exists is perfectly 

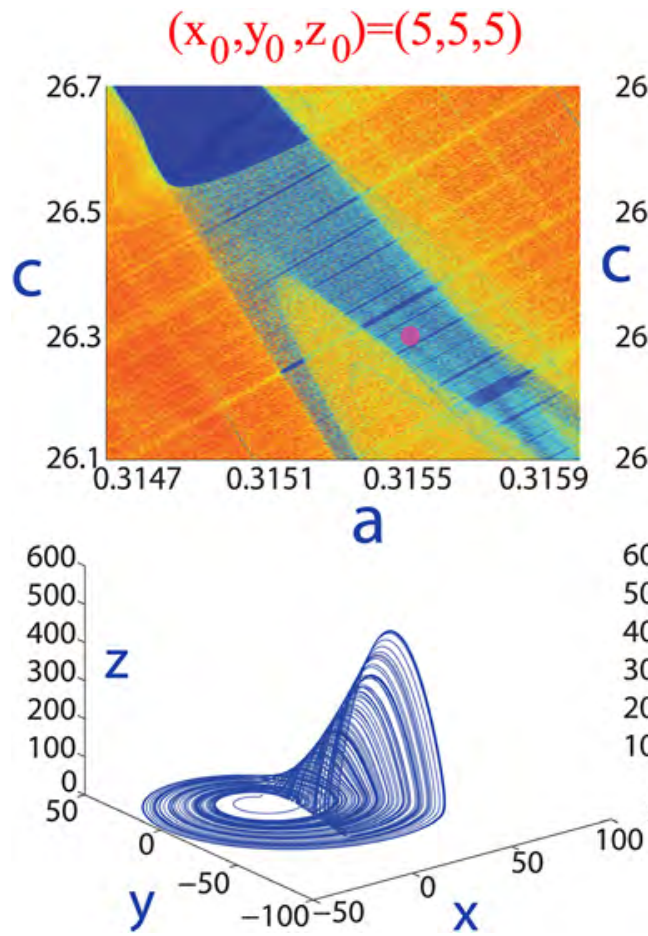

located (in green in top pictures) by the chaos indicator technique. This chaotic attractor and the stable periodic orbits are bounded. Although in the dominant unbounded motion region, their basin of attraction is fractal, the attractors have

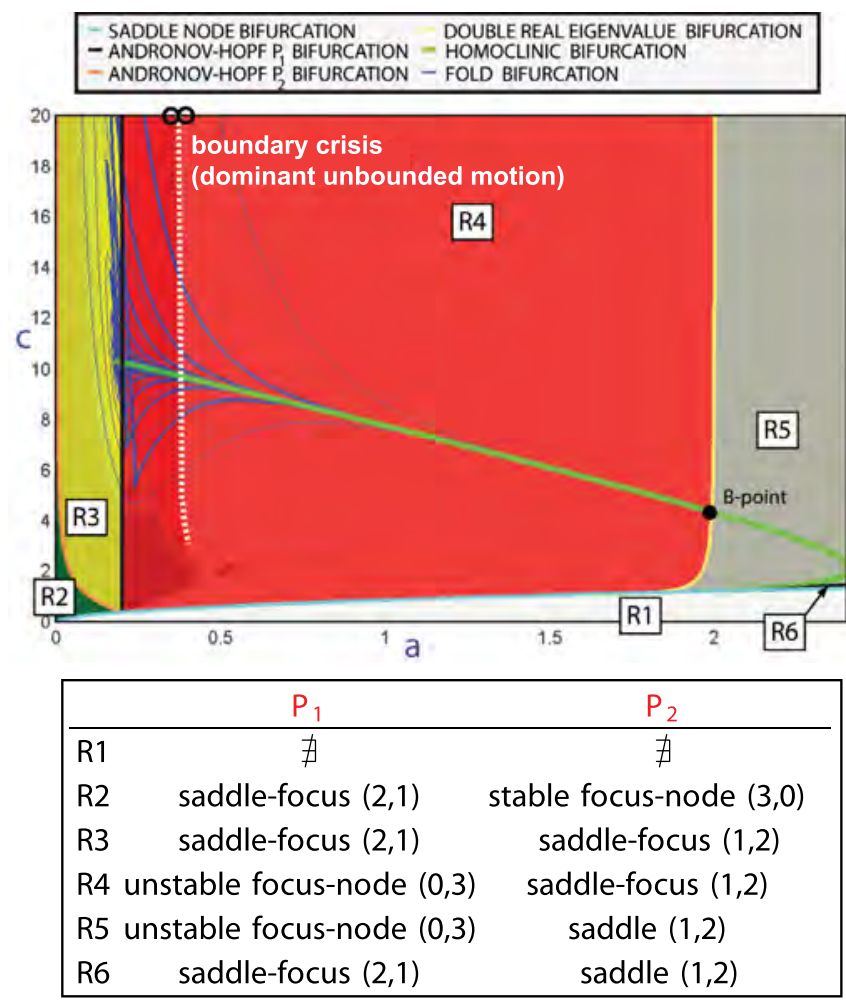

FIG. 8. Regions R1-R6 with different type of equilibrium points (for $b=0.2)$ and some related bifurcations. The numbers in brackets denote the dimension of the stable and unstable manifolds of the equilibria $P_{1,2}$. The boundary crisis that generates dominant unbounded dynamics occurs in region R4, where the equilibrium point $P_{1}$, which organizes the unbounded dynamics, is an unstable focus-node. the same structure on both sides of the crisis. The only difference appears below the interior crisis, where we have a "thick" chaotic attractor (A1) or unbounded dynamics after a transient chaos (B1). These changes may be seen in the pictures on the middle, where we show the maximum Lyapunov exponent (MLE) in two vertical segments.

Therefore, the global structure of the parametric space is the same all over, the difference appears when the "thin"
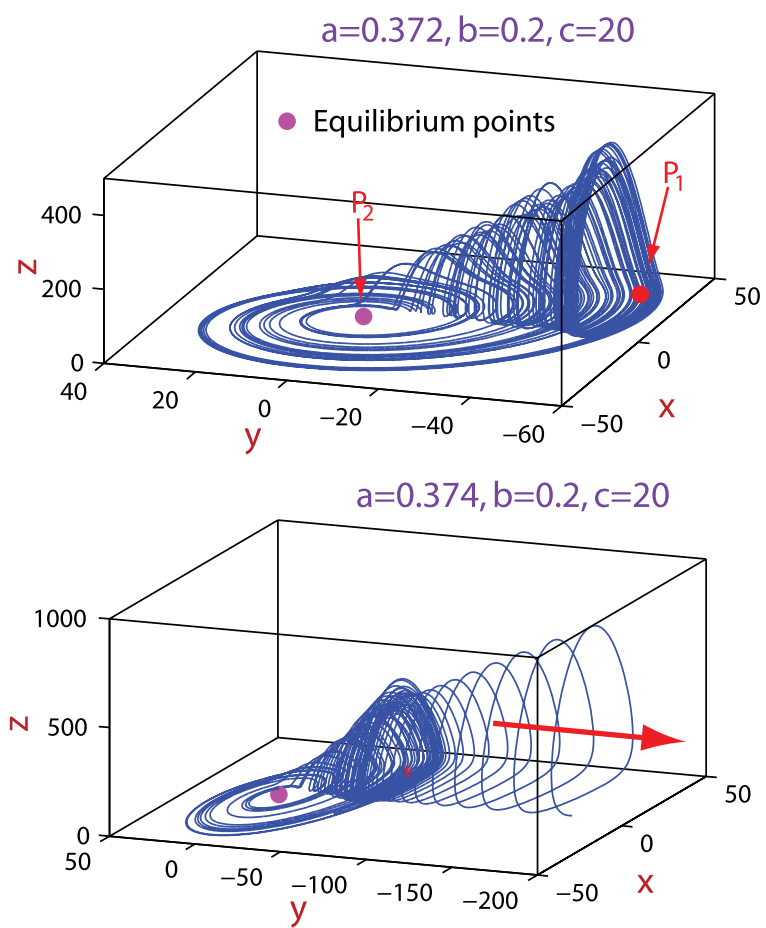

FIG. 9. Bounded and unbounded behavior for two orbits with the same initial conditions but slightly different sets of parameter values (before and after the boundary crisis, respectively). 


\section{Chaotic attractor or unbounded orbit}

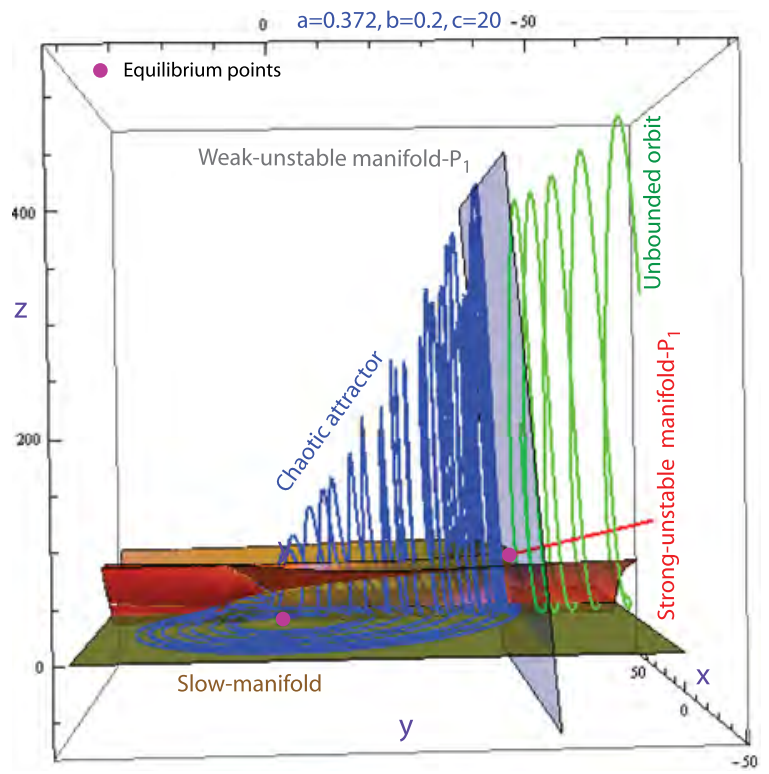

Transient chaos - unbounded orbit

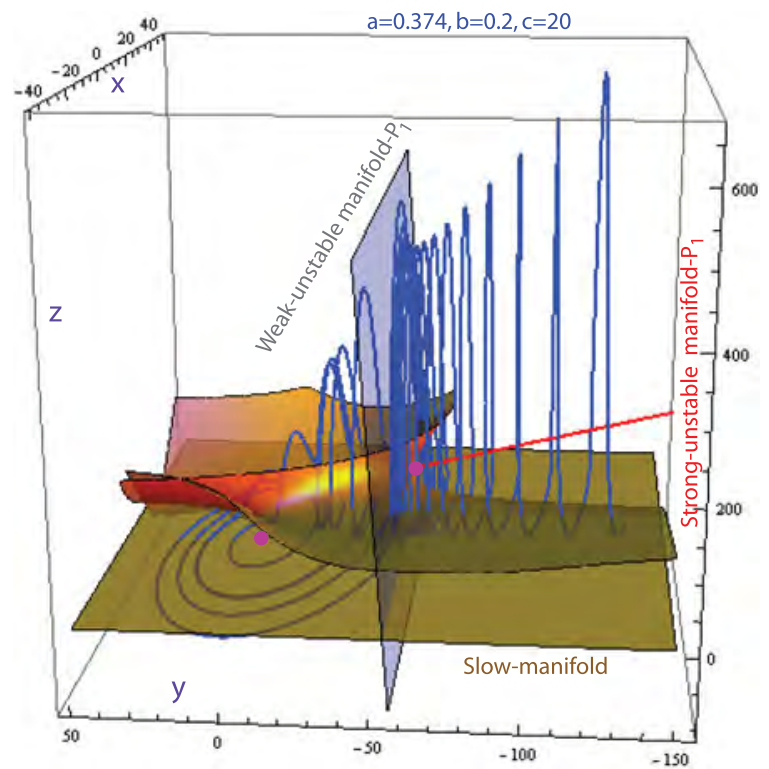

FIG. 10. Explanation of the origin of the dominant unbounded behavior: the change in the relative position of the chaotic invariant set and the weakunstable manifold.

attractor experiences a crisis. What really happens in this situation is that there is a crossing point of two branches, the interior crisis and the boundary crisis. This crossing point is called double crisis point in literature, ${ }^{24,25}$ and at that point, both crisis bifurcations change their nature. In Fig. 12, on the left, we show a schematic diagram of the change of dynamics. ${ }^{25}$ In the bottom region (below the horizontal line), from left to right, the big chaotic attractor experiences a boundary crisis. However, in the top region, the attractor does not experience any change in shape or stability. The only change takes place in the size and shape of the basin of attraction, which now becomes fractal (we have crossed a basin metamorphosis bifurcation), as shown in Subsection III A in Fig. 6. To the left of the vertical line, if we move from top to bottom, the chaotic attractor experiences an interior crisis becoming wider. In contrast, to the right of the vertical line, the attractor undergoes a boundary crisis. The right picture of Fig. 12 shows the location of one double crisis point in a BPD diagram, which includes both behaviors shown in Fig. 11 . This picture presents all the changes shown in the schematic diagram. ${ }^{25}$

Note that the above mechanism can be also applied to the case of having a saddle-focus equilibrium point with a dominant unstable manifold and a weak stable manifold (focus). What is important is the existence of the boundary given by the weak manifold, given by the complex eigenvalues of the focus, and a dominant unstable manifold that leads to the unbounded motion (of course it is also necessary to have a positive divergence in unbounded regions of the phase space).

\section{RÖSSLER MODEL: SLOW MANIFOLDS AND UNBOUNDED DYNAMICS}

In the previous sections, we have studied the unbounded dynamics from a numerical point of view. It remains to study which is the behavior when the different parameters grow. In this situation, the Fenichel's theory provides us information about transient behaviors of the orbits and alternative routes to escape.

First, we briefly review some basic results on singular perturbation theory (see Refs. 26-28 for theoretical settings and Refs. 29 and 30 for applications).

Consider an autonomous system written on the form (slow-fast system on slow-time formulation)

$$
\begin{aligned}
\dot{\mathbf{x}} & =f(\mathbf{x}, \mathbf{y} ; \varepsilon)=f_{0}(\mathbf{x}, \mathbf{y})+\varepsilon \ldots, \quad \mathbf{x} \in D \subset \mathbb{R}^{n}, \\
\varepsilon \dot{\mathbf{y}} & =g(\mathbf{x}, \mathbf{y} ; \varepsilon)=g_{0}(\mathbf{x}, \mathbf{y})+\varepsilon \ldots, \quad \mathbf{y} \in G \subset \mathbb{R}^{m},
\end{aligned}
$$

where $\varepsilon$ is a small parameter. We assume that $f$ and $g$, as well as all their derivatives, are $\mathcal{O}(1)$ as $\varepsilon \downarrow 0$. In this system, $\mathbf{x}$ and $\mathbf{y}$ are called the slow and fast variables, respectively. In the limit case $\varepsilon=0$, the system becomes the DAE system (reduced slow system)

$$
\dot{\mathbf{x}}=f(\mathbf{x}, \mathbf{y} ; 0)=f_{0}(\mathbf{x}, \mathbf{y}), \quad 0=g(\mathbf{x}, \mathbf{y} ; 0)=g_{0}(\mathbf{x}, \mathbf{y}) .
$$

The zero set of $g(\mathbf{x}, \mathbf{y} ; 0)$ defines a manifold $\mathcal{M}_{0}$ in phase space, the so called critical manifold to which the motion of the reduced slow system is confined. We focus on systems for which the critical manifold is represented by the graph of a function, which is defined on a compact domain $D \equiv K_{\mathbf{x}} \in \mathbb{R}^{n}$, that is, $\mathcal{M}_{0}=\left\{(\mathbf{x}, \mathbf{y}) \in \mathbb{R}^{n+m} \mid \mathbf{y}=h_{0}(\mathbf{x}), \mathbf{x} \in K_{\mathbf{x}}\right\}$, and which are normally hyperbolic. A manifold $\mathcal{M}$ is called normally hyperbolic if the normal bundle of $\mathcal{M}$ can be split with respect to the flow generated by the dynamical system in an exponentially stable one, $N^{s}$, and an exponentially unstable one, $N^{u}$ (that is, it is hyperbolic) and if $N^{s}$ and $N^{u}$ contract and expand more sharply than the tangent bundle $T(\mathcal{M})$ under the flow.

The question of whether ${ }^{28}$ the slow manifold $\mathcal{M}_{\varepsilon}$ approximated by $\mathcal{M}_{0}$ persists for $\varepsilon>0$ was answered by Fenichel. ${ }^{26}$ Who stated that if $\mathcal{M}_{0}$ is a compact manifold that is normally hyperbolic, then there exists, for sufficiently small, positive $\varepsilon$, a smooth manifold $\mathcal{M}_{\varepsilon}$ close to $\mathcal{M}_{0}$.

Now we may state the following result when $a \uparrow+\infty$, $c \uparrow+\infty$ or both $a, c \uparrow+\infty$ or $b, c \uparrow+\infty$ (the Fenichel's 

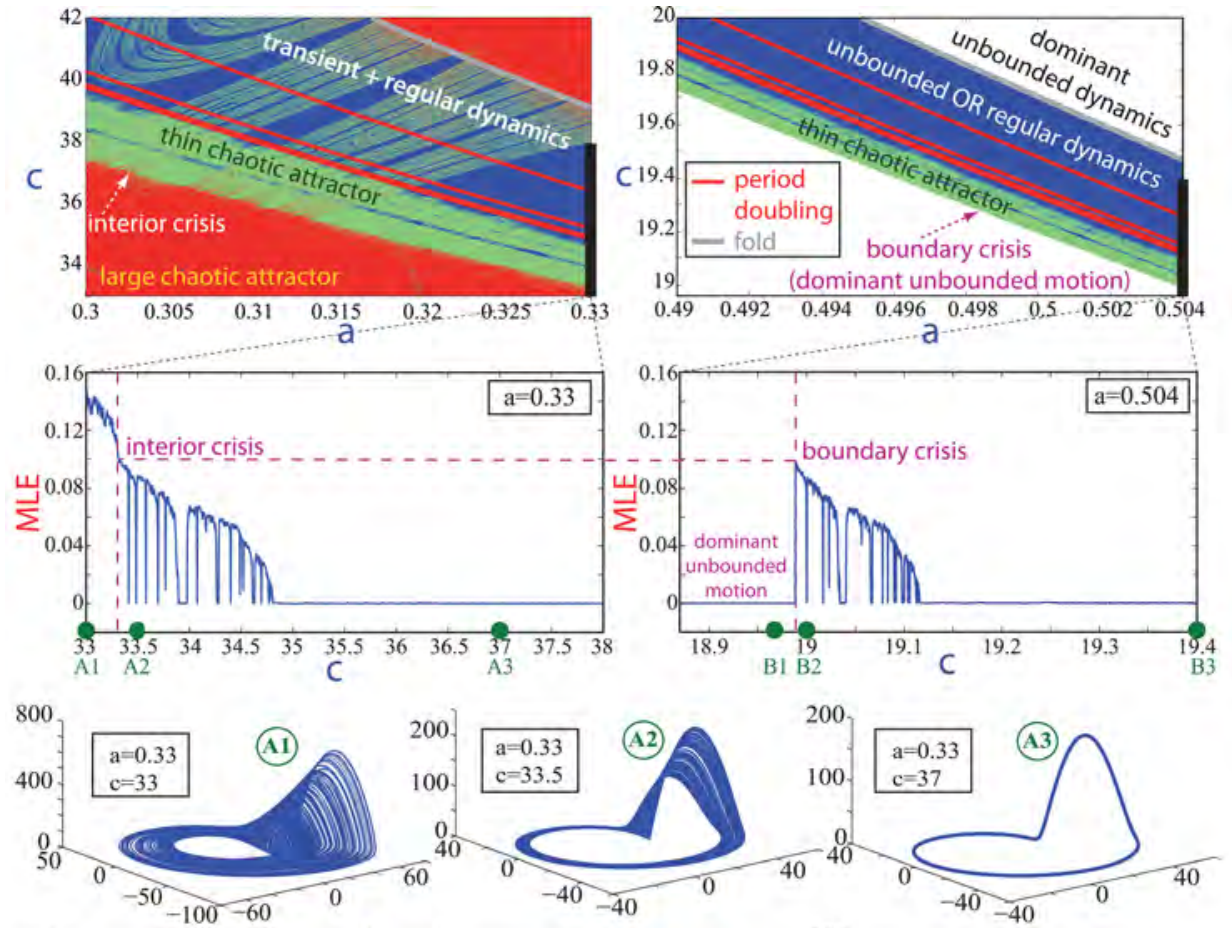

FIG. 11. Top: BPD of two different parametric regions, before and after the studied boundary crisis, respectively. Second row: MLE for two vertical segments. Third and Fourth rows: Similar trajectories for both regions except $A 1$ and $B 1$ with bounded and unbounded behavior, respectively.
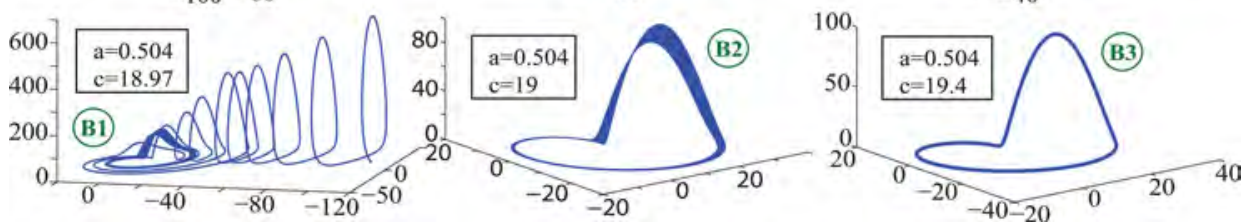

theory cannot be applied to the rest of the cases $b \uparrow+\infty$ and $a, b \uparrow+\infty$ as in those cases the system does not fit the slowfast formulation (2)). Note that we have to restrict ourselves to work on a compact domain $K_{\mathbf{x}}$, although the Rössler system may be unbounded, and so the validity of the result is just on the initial stages of the unbounded behavior.

Theorem. For any sufficiently small $\varepsilon$ defined as $\varepsilon=a^{-1}$, $\varepsilon=c^{-1}, \quad \varepsilon=a^{-1}=(\lambda c)^{-1}\left(\lambda \in \mathbb{R}^{+}\right)$or $\varepsilon=b^{-1}=(\lambda c)^{-1}$ $\left(\lambda \in \mathbb{R}^{+}\right)$, there is a function $h_{\varepsilon}$ such that the graph

$$
\mathcal{M}_{\varepsilon}=\left\{(\mathbf{x}, \mathbf{y}) \mid \mathbf{y}=h_{\varepsilon}(\mathbf{x}), \mathbf{x} \in K_{\mathbf{x}}\right\}
$$

is locally invariant under the dynamics of the Rössler equations (1). The function $h_{\varepsilon}$ admits an asymptotic expansion on the compact set $K_{\mathbf{x}}$

$$
h_{\varepsilon}(\mathbf{x})=h_{0}(\mathbf{x})+\varepsilon h_{1}(\mathbf{x})+\varepsilon^{2} h_{2}(\mathbf{x})+\cdots \quad \text { as } \quad \varepsilon \downarrow 0,
$$

where the coefficients $h_{i}: K_{\mathbf{x}} \subset \mathbb{R}^{n} \rightarrow \mathbb{R}^{m}$ are found successively by using the equations

(1) In case $\varepsilon=a^{-1}$, the fast variable is $\mathbf{y}=y$ and the equations of the slow manifold $\mathcal{M}_{\varepsilon}^{a}$ are given by

$$
\begin{aligned}
h_{0} & =0, \quad h_{1}=-x \\
h_{i+1}(x, z) & =-z \frac{\partial h_{i}}{\partial x}+(b+z(x-c)) \frac{\partial h_{i}}{\partial z}-\sum_{j=0}^{i} h_{j} \frac{\partial h_{i-j}}{\partial x} .
\end{aligned}
$$

(2) In case $\varepsilon=c^{-1}$, the fast variable is $\mathbf{y}=z$ and the equations of the slow manifold $\mathcal{M}_{\varepsilon}^{c}$ are given by

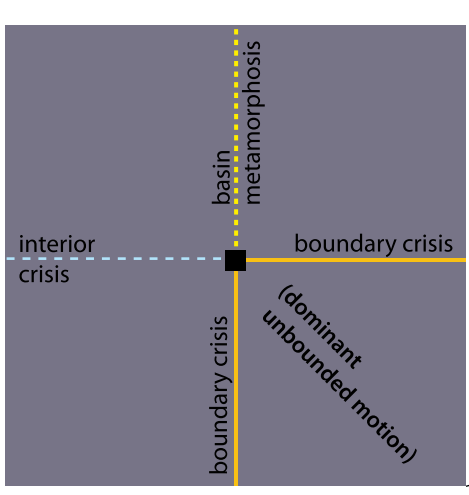

Schematic diagram

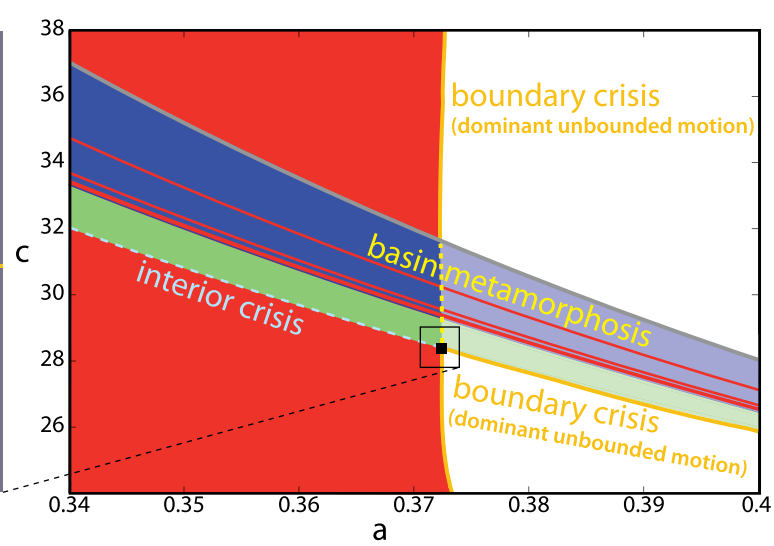

FIG. 12. Double crisis point originated by the intersection of an interior crisis and a boundary crisis. On the left, an schematic diagram; and on the right, an example taken from a specific band in the Rössler model. 


$$
\begin{aligned}
h_{0} & =0, \quad h_{1}=b, \\
h_{i+1}(x, y) & =x h_{i}+y \frac{\partial h_{i}}{\partial x}-(x+a y) \frac{\partial h_{i}}{\partial y}+\sum_{j=0}^{i} h_{j} \frac{\partial h_{i-j}}{\partial x} .
\end{aligned}
$$

(3) In case $\varepsilon=b^{-1}=(\lambda c)^{-1}$, the fast variable is $\mathbf{y}=z$ and the equations of the slow manifold $\mathcal{M}_{\varepsilon}^{b c}$ are given by

$$
\begin{aligned}
h_{0} & =\lambda, \\
h_{i+1}(x, y) & =x h_{i}+y \frac{\partial h_{i}}{\partial x}-(x+a y) \frac{\partial h_{i}}{\partial y}+\sum_{j=0}^{i} h_{j} \frac{\partial h_{i-j}}{\partial x} .
\end{aligned}
$$

(4) In case $\varepsilon=a^{-1}=(\lambda c)^{-1}$, the fast variables are $\mathbf{y}=(y, z)$ and the equations of the slow manifold $\mathcal{M}_{\varepsilon}^{a c}$ are given by

$$
\begin{aligned}
h_{0}^{y} & =0, \quad h_{1}^{y}(x)=-\frac{x}{\lambda}, \\
h_{i+1}^{y}(x) & =-\frac{1}{\lambda} \sum_{j=0}^{i}\left(h_{j}^{y}+h_{j}^{z}\right) \frac{\mathrm{d} h_{i-j}^{y}}{\mathrm{~d} x}, \\
h_{0}^{z} & =0, \quad h_{1}^{z}=b, \\
h_{i+1}^{z}(x) & =x h_{i}^{z}+\sum_{j=0}^{i}\left(h_{j}^{y}+h_{j}^{z}\right) \frac{\mathrm{d} h_{i-j}^{z}}{\mathrm{~d} x} .
\end{aligned}
$$

Furthermore, $h_{\varepsilon} \in \mathcal{C}^{r}$ for any finite $r$, and it is attracting in cases $\varepsilon=c^{-1}$ and $\varepsilon=b^{-1}=(\lambda c)^{-1}\left(\lambda \in \mathbb{R}^{+}\right)$; repulsive in case $\varepsilon=a^{-1}$ and it has attractive and repulsive directions in case $\varepsilon=a^{-1}=(\lambda c)^{-1}\left(\lambda \in \mathbb{R}^{+}\right)$.

Proof. The theorem is based on Fenichel's theory. ${ }^{27}$ We just have to prove the basic requirements of the theory, that is, that the critical manifold is normally hyperbolic. As in the four cases, the analysis is quite similar, we just detail the case $\varepsilon=a^{-1}=(\lambda c)^{-1}\left(\lambda \in \mathbb{R}^{+}\right)$. Now, the slow-time system is given by

$$
\dot{x}=-(y+z), \quad \varepsilon \dot{y}=\varepsilon x+\lambda y, \quad \varepsilon \dot{z}=\varepsilon b+\varepsilon x z-z .
$$

So, $\mathcal{M}_{0}=\{(x, y, z) \mid y=0, z=0\}$, the fast variables are $\mathbf{y}=(y, z)$, and

$$
\left.g_{\mathbf{y}}\left(\mathbf{x}, h_{0} ; 0\right) \equiv \frac{\partial(\varepsilon x+\lambda y, \varepsilon b+\varepsilon x z-z)}{\partial(y, z)}\right|_{\mathcal{M}_{0}}=\left(\begin{array}{cc}
\lambda & 0 \\
0 & -1
\end{array}\right) .
$$

Therefore, $\Re\left\{\operatorname{Sp}\left(g_{\mathbf{y}}\left(\mathbf{x}, h_{0} ; 0\right)\right)\right\}=\{-1, \lambda\}$ (with $\Re \mathrm{Sp}$ the real part of the spectrum) and the normal bundle has one dimensional exponentially stable and unstable parts. So, the manifold $\mathcal{M}_{0}$ is normally hyperbolic but it has attractive and repulsive directions. Now, from the Fenichel's theorems, there is a function $h_{\varepsilon}$ such that its graph on the compact set $K_{\mathbf{x}}$ defines the slow manifold $\mathcal{M}_{\varepsilon}^{a c}$. The function $h_{\varepsilon} \equiv$ $\left(h_{\varepsilon}^{y}, h_{\varepsilon}^{z}\right)$ admits an asymptotic expansion

$$
h_{\varepsilon}(x)=h_{0}(x)+\varepsilon h_{1}(x)+\varepsilon^{2} h_{2}(x)+\cdots \quad \text { as } \quad \varepsilon \downarrow 0,
$$

where the coefficients $h_{i} \equiv\left(h_{i}^{y}, h_{i}^{z}\right): K_{\mathbf{x}} \subset \mathbb{R} \rightarrow \mathbb{R}^{2}$ are found successively by using the equations of the fast variables $\varepsilon(\dot{y}, \dot{z})=(\varepsilon x+\lambda y, \varepsilon b+\varepsilon x z-z)$. That is, by substitution $y=h_{\varepsilon}^{y}(x)$ and $z=h_{\varepsilon}^{z}(x)$, we have the invariance equations

$$
\begin{aligned}
& \varepsilon\left(D h_{\varepsilon}^{y}\right)(x)\left(-h_{\varepsilon}^{y}(x)-h_{\varepsilon}^{z}(x)\right)=\varepsilon x+\lambda h_{\varepsilon}^{y}(x), \\
& \varepsilon\left(D h_{\varepsilon}^{z}\right)(x)\left(-h_{\varepsilon}^{y}(x)-h_{\varepsilon}^{z}(x)\right)=\varepsilon b+\varepsilon x h_{\varepsilon}^{z}(x)-h_{\varepsilon}^{z}(x) .
\end{aligned}
$$

Now, by taking coefficients of the same degree on the small parameter $\varepsilon$, we obtain the formula for the terms $h_{i}^{y}(x)$ and $h_{i}^{z}(x)$.

Just to give an idea of the equations of the slow manifolds, we give explicit expressions of them up to third order in $\varepsilon$

$$
\begin{aligned}
\mathcal{M}_{\varepsilon}^{a}= & \left\{(x, y, z) \mid y=-\varepsilon x+\varepsilon^{2} z+\varepsilon^{3}(b-x+z(x-c))\right) \\
& \left.+\mathcal{O}\left(\varepsilon^{4}\right)\right\}, \\
\mathcal{M}_{\varepsilon}^{c}= & \left\{(x, y, z) \mid z=b\left(\varepsilon+\varepsilon^{2} x+\varepsilon^{3}\left(x^{2}+y\right)\right)+\mathcal{O}\left(\varepsilon^{4}\right)\right\}, \\
\mathcal{M}_{\varepsilon}^{b c}= & \left\{(x, y, z) \mid z=\lambda\left(1+\varepsilon x+\varepsilon^{2}\left(y+\lambda+x^{2}\right)\right.\right. \\
& \left.\left.+\varepsilon^{3}\left(x^{3}-a y+x(1+4 \lambda+3 y)\right)\right)+\mathcal{O}\left(\varepsilon^{4}\right)\right\}, \\
\mathcal{M}_{\varepsilon}^{a c}= & \left\{(x, y, z) \mid y=-\varepsilon \frac{x}{\lambda}-\varepsilon^{3} \frac{1}{\lambda^{2}}\left(-\frac{x}{\lambda}+b\right)+\mathcal{O}\left(\varepsilon^{4}\right),\right. \\
z= & \left.b\left(\varepsilon+\varepsilon^{2} x+\varepsilon^{3} x^{2}\right)+\mathcal{O}\left(\varepsilon^{4}\right)\right\} .
\end{aligned}
$$

Note that the theorem requires that in the case when both $a$ or $b$ and $c$ tend to infinity, they have a linear relation $a$ or $b=\lambda c$, but this is just a technical hypothesis to give an explicit expression of the slow manifold in such a case. To use Fenichel's theorem; and therefore to justify the existence of the manifold in this case, we just have to impose that both tend to infinity.

In Fig. 13, we show the evolution of four orbits in cases $c, a, b=c$ or $a=c$ large and the corresponding slow manifolds $\mathcal{M}_{\varepsilon}$. To be precise, the complete set of values of the parameters are $(a, b, c)=(0.4,1,1000),(100,1,1),(0.4,1000$, $1000)$, or $(100,1,100)$, respectively. The big green dot shows the initial conditions $\left(x_{0}, y_{0}, z_{0}\right)$ and the red square the final point of the orbit; whereas on grey, we show the attracting manifolds and in pink the non-attracting ones.

In case $a \uparrow \infty$, the slow manifold is repulsive and the orbits go out of the manifold exponentially fast ${ }^{28}$ and in case $a=c \uparrow \infty$, the slow manifold has attracting and repulsive directions. Note that this behavior is on a compact domain $K_{\mathbf{x}}$ and when the variables go away, due to the unbounded behavior, the theory just gives some indications
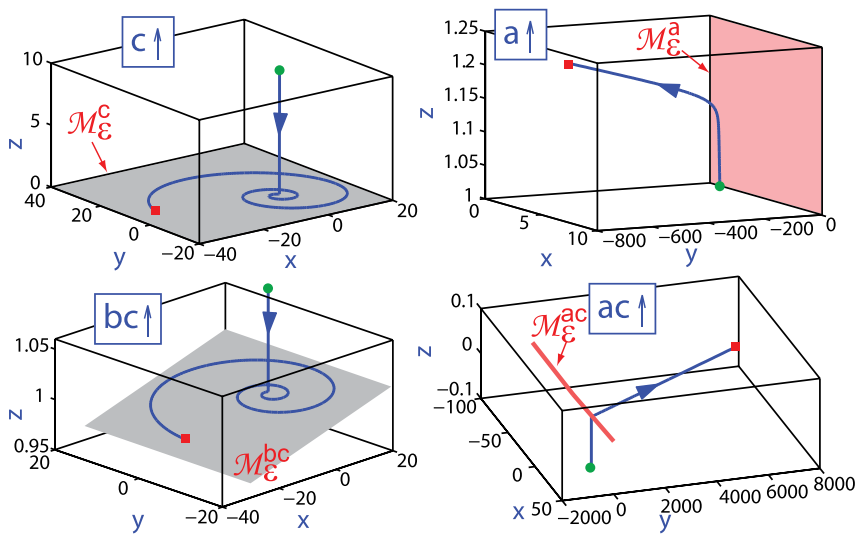

FIG. 13. Slow manifolds and an orbit approaching (left) or going far away (right) from them, when $a \uparrow+\infty, c \uparrow+\infty$ or both $a, c \uparrow+\infty$ or $b, c \uparrow+\infty$. 
$\left(x_{0}, y_{0}, z_{0}\right)=(1,1,10)$

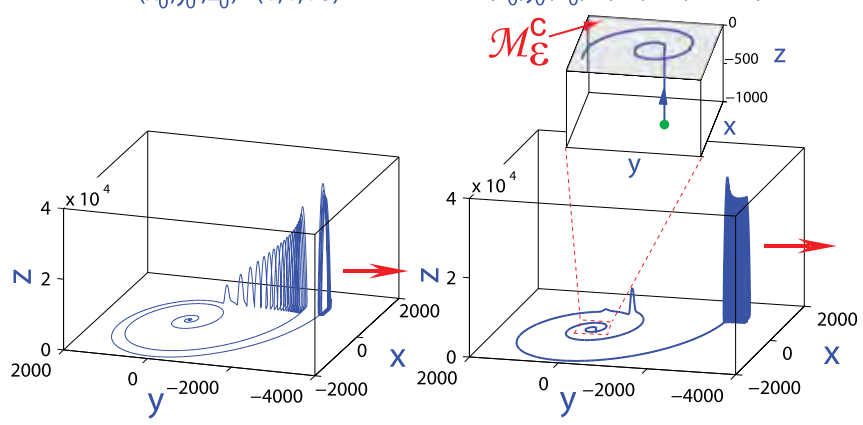

FIG. 14. Long time evolution of two orbits in a case of attracting slow manifold $((a, b, c)=(0.4,1,1000))$.

on the way of going out. In any case, this gives us an extra escaping mechanism, due to the impulse given by the repulsive manifolds or directions. This can be seen in the right pictures of Fig. 13.

In cases $c \uparrow \infty$ and $b=\lambda c \uparrow \infty$, the slow manifold $\mathcal{M}_{\varepsilon}$ is attracting. This fact has a great influence in the transient dynamics of the orbits, as they go initially to the attracting slow manifold, staying close to it during a transient time as shown in left pictures of Fig. 13. If the orbits cross the two-dimensional weak unstable (stable) manifold of $P_{1}$, then they continue the unbounded dynamics following the leading unstable direction, as explained in the previous sections. An example of this situation is presented in Fig. 14, where we show on the left the same orbit $\left(\left(x_{0}, y_{0}, z_{0}\right)=(1,1,10)\right)$ as in case $c$ large $((a, b, c)=(0.4,1,1000))$ of Fig. 13, but for longer integration time, and on the right for a different set of initial conditions $\left(\left(x_{0}, y_{0}, z_{0}\right)=(10,-70,-1000)\right)$ (small picture for short integration time and large picture for longer integration time). Note that the final dynamics of the orbits follow the leading unstable manifold of $P_{1}$ denoted by a red arrow.

From the figures, we observe that the slow manifold theory has a role in the evolution of the first stages of the orbit for large values of the parameters, giving the transient dynamics in the attracting slow manifold or providing fast escapes in the repulsive case.

\section{CONCLUSIONS}

We have studied in detail the mechanism that creates large regions of dominant unbounded dynamics in the wellknown Rössler model. Unbounded dynamics appears, mainly, when the divergence of the flow is positive in unbounded regions in phase space; but in any case, it can be the dominant behavior or not when there are also large regions with negative divergence. Therefore, to grasp the mechanism that creates this dominant behavior is an important goal to improve understanding of the dynamics of these flows. Several numerical tests have been done using several state-of-the-art numerical methods to study dynamical systems, as chaos indicators, basins of attraction, and bifurcation analysis. This combined use of several techniques of different nature provides a complete study.

The bifurcation skeleton of the system provides relevant information, as around the bifurcation lines small regions of bounded motion persist. Moreover, the numerical study using chaos indicators also gives information in the whole parametric space. We have observed that along the complete parametric space the global bifurcation curves are maintained. The difference is that, after the boundary crisis that leads to a dominant unbounded dynamics, there is no more a "thick" chaotic attractor but a transient chaotic dynamics instead. At the same time, the basins of attraction of the bounded attractors become fractal. Besides, double crisis points are located in the intersection of this boundary crisis and the interior crises of the chaotic attractor. For very large values of the parameters, the Rössler system has been studied by means of Fenichel's theory, providing formulas for computing the slow manifolds which give information concerning the transient dynamics of the orbits and giving alternative routes of escape.

Our results in the Rössler model permit to describe a particular case of boundary crisis related to the point where the unbounded dynamics becomes the dominant one in this system. This crisis occurs in flows with an unstable focusnode (or a saddle-focus) equilibrium point and a chaotic invariant set (saddle or attractor) on the system. When the weak-unstable (or weak-stable) manifold of this equilibrium point is tangent to the chaotic invariant set, the crisis arises. These results fit well with previous studies on maps and flows in the literature, giving a generic framework.

\section{ACKNOWLEDGMENTS}

This work was supported by Spanish Research project MTM2012-31883. The authors thank the suggestions and remarks of the referees that have contributed to improve the paper.

${ }^{1}$ B. Eckhardt and C. Jung, "Regular and irregular potential scattering," J. Phys. A 19, L829-L833 (1986).

${ }^{2}$ Y.-C. Lai and T. Tél, "Complex dynamics on finite-time scales," Transient Chaos, Applied Mathematical Sciences Vol. 173 (Springer, New York, 2011), pp. xiv +497 .

${ }^{3}$ J. M. Seoane and M. A. F. Sanjuán, "New developments in classical chaotic scattering," Rep. Prog. Phys. 76, 016001 (2013).

${ }^{4}$ T. Tél and M. Gruiz, Chaotic Dynamics: An Introduction Based on Classical Mechanics (Cambridge University Press, 2006).

${ }^{5}$ C. Grebogi, E. Ott, and J. A. Yorke, "Chaotic attractors in crisis," Phys. Rev. Lett. 48, 1507 (1982).

${ }^{6}$ C. Grebogi, E. Ott, and J. A. Yorke, "Crises, sudden changes in chaotic attractors, and transient chaos," Physica D 7, 181-200 (1983).

${ }^{7}$ R. Barrio, "Sensitivity tools vs. Poincaré sections," Chaos Solitons Fractals 25, 711-726 (2005).

${ }^{8}$ R. Barrio, "Painting chaos: A gallery of sensitivity plots of classical problems,” Int. J. Bifur. Chaos 16, 2777-2798 (2006).

${ }^{9}$ O. E. Rössler, "An equation for continuous chaos," Phys. Lett. A 57, 397-398 (1976)

${ }^{10} \mathrm{P}$. Collet and J.-P. Eckmann, Iterated Maps on the Interval as Dynamical Systems, Progress in Physics Vol. 1 (Birkhäuser, Boston, Mass., 1980).

${ }^{11}$ M. Hénon, "A two-dimensional mapping with a strange attractor," Commun. Math. Phys. 50, 69-77 (1976).

${ }^{12}$ C. Simó, "On the Hénon-Pomeau attractor,” J. Stat. Phys. 21, 465-494 (1979).

${ }^{13}$ R. Barrio, F. Blesa, and S. Serrano, "Qualitative analysis of the Rössler equations: Bifurcations of limit cycles and chaotic attractors," Phys. D 238, 1087-1100 (2009).

${ }^{14}$ R. Barrio, F. Blesa, S. Serrano, and A. Shilnikov, "Global organization of spiral structures in biparameter space of dissipative systems with Shilnikov saddle-foci," Phys. Rev. E 84, 035201 (2011).

${ }^{15}$ R. Barrio, F. Blesa, and S. Serrano, "Topological changes in periodicity hubs of dissipative systems,” Phys. Rev. Lett. 108, 214102 (2012). 
${ }^{16}$ R. Genesio, G. Innocenti, and F. Gualdani, "A global qualitative view of bifurcations and dynamics in the Rössler system," Phys. Lett. A 372, 1799-1809 (2008).

${ }^{17}$ L. Gardini, "Hopf bifurcations and period-doubling transitions in Rössler model," Nuovo Cimento B 89(11), 139-160 (1985).

${ }^{18}$ E. J. Doedel, R. C. Paffenroth, A. R. Champneys, T. F. Fairgrieve, Y. A. Kuznetsov, B. E. Oldeman, B. Sandstede, and X. J. Wang, Auto2000: Continuation and Bifurcation Software for Ordinary Differential Equations (2000).

${ }^{19}$ L. A. Belyakov, "Bifurcation set in a system with homoclinic saddle curve," Math. Notes USSR Acad. Sci. 28(6), 910-916 (1980).

${ }^{20}$ A. R. Champneys and Y. A. Kuznetsov, "Numerical detection and continuation of codimension-two homoclinic bifurcations," Int. J. Bifur. Chaos 4, 785-822 (1994).

${ }^{21}$ V. Castro, M. Monti, W. B. Pardo, J. A. Walkenstein, and E. Rosa, Jr., "Characterization of the Rössler system in parameter space," Int. J. Bifurcation Chaos 17, 965-973 (2007).

${ }^{22}$ Y. A. Kuznetsov, Elements of Applied Bifurcation Theory, Applied Mathematical Sciences Vol. 112, 3rd ed. (Springer-Verlag, New York, 2004).
${ }^{23}$ J.-M. Ginoux, B. Rossetto, and L. O. Chua, "Slow invariant manifolds as curvature of the flow of dynamical systems," Int. J. Bifurcation Chaos $\mathbf{1 8}$, 3409-3430 (2008).

${ }^{24}$ H. Stewart, J. Thompson, Y. Ueda, and A. Lansbury, "Optimal escape from potential wells-patterns of regular and chaotic bifurcation," Physica D 85, 259-295 (1995).

${ }^{25}$ H. B. Stewart, Y. Ueda, C. Grebogi, and J. A. Yorke, "Double crises in two-parameter dynamical systems," Phys. Rev. Lett. 75, 2478-2481 (1995).

${ }^{26} \mathrm{~N}$. Fenichel, "Geometric singular perturbation theory for ordinary differential equations," J. Differential Equations 31, 53-98 (1979).

${ }^{27}$ C. K. R. T. Jones, "Geometric singular perturbation theory," Lecture Notes Math. 1609, 44-118 (1995).

${ }^{28} \mathrm{~F}$. Verhulst, Methods and Applications of Singular Perturbations, Texts in Applied Mathematics Vol. 50 (Springer, New York, 2005).

${ }^{29}$ J. Guckenheimer, K. Hoffman, and W. Weckesser, "The forced van der Pol equation. I. The slow flow and its bifurcations," SIAM J. Appl. Dyn. Syst. 2, 1-35 (2003).

${ }^{30}$ H. G. Kaper and T. J. Kaper, "Asymptotic analysis of two reduction methods for systems of chemical reactions," Phys. D 165, 66-93 (2002). 